\title{
Late-time evolution of a self-interacting scalar field in the spacetime of dilaton black hole
}

\author{
Rafał Moderski \\ Nicolaus Copernicus Astronomical Center \\ Polish Academy of Sciences \\ 00-716 Warsaw, Bartycka 18, Poland \\ moderski@camk.edu.pl \\ Marek Rogatko \\ Technical University of Lublin \\ 20-618 Lublin, Nadbystrzycka 40, Poland \\ rogat@tytan.umcs.lublin.pl \\ rogat@akropolis.pol.lublin.pl
}

(October 26, 2018)

\begin{abstract}
We investigate the late-time tails of self-interacting (massive) scalar fields in the spacetime of dilaton black hole. Following the no hair theorem we examine the mechanism by which self-interacting scalar hair decay. We revealed that the intermediate asymptotic behavior of the considered field perturbations is dominated by an oscillatory inverse power-law decaying tail. The numerical simulations showed that at the very late-time massive self-interacting scalar hair decayed slower than any power law.
\end{abstract}

PACS numbers: 04.50.+h, 98.80.Cq

\section{INTRODUCTION}

The late-time evolution of various fields outside a collapsing body plays an important role in major aspects of black hole physics, as the no-hair theorem [1] and the mass inflation scenario [2]. Wheeler coined the metaphoric dictum black holes have no hair which means that regardless of the specific details of the collapse or structure and properties of the collapsing body a stationary black hole characterized by its mass, charge and angular momentum emerged in the resultant process. Therefore it is interesting to study the mechanism responsible for the decay of black hole hair. The neutral external perturbations were first studied in [3] and it was found that the late-time behavior is dominated by the factor $t^{-(2 l+3)}$, for each multipole moment $l$. The neutral perturbations along null infinity and along the future event horizon were studied in Ref. [4]. Their decays were governed by the power laws $u^{-(l+2)}$ and $v^{-(l+3)}$, where $u$ and $v$ were the outgoing Eddington-Filkenstein (ED) and ingoing ED coordinates. Bicak [5] considered the scalar perturbations on Reissner-Nordtröm (RN) background and found that for $|Q|<M$ the relation $t^{-(2 l+2)}$, while for $|Q|=M$ the late-time behavior at fixed $r$ was governed by $t^{-(l+2)}$.

Hod and Piran [6]- 8] studied the late time behavior of a charged massless scalar field in RN spacetime. Among all the conclusion was that a charged hair decayed slower than a neutral one.

The problem of late-time tails in gravitational collapse of a self-interacting (SI) fields in the background of Schwarzschild solution was reported by Burko [9] and in RN spacetime, at intermediate late-time, was considered in Ref. [10]. At intermediate late-time for small mass $m$ the decay was dominated by the oscillatory inverse power tails $t^{-(l+3 / 2)} \sin (m t)$. This analytic prediction was verified at intermediate times, where $m M \leq m t \leq 1 /(m M)^{2}$. In Ref. 111] the nearly extreme RN spacetime was considered and it was found analytically that the inverse power-law 
behavior of the dominant asymptotic tail is of the form $t^{-5 / 6} \sin (m t)$, independent of $l$. The asymptotic tail behavior of SI scalar field was also studied in Schwarzschild spacetime [12]. The oscillatory tail of scalar field has the decay rate of $t^{-5 / 6}$ at asymptotically late time.

Nowadays, it seems that the superstring theories are the most promising candidates for a consistent quantum theory of gravitation. In Ref. [13] the asymptotic evolution of a massless charged scalar field in the background of dilaton black hole was studied. Using both numerical and analytical methods the inverse power-law relaxation was revealed. It turned out that the charged hair decayed slower than a neutral one.

In our work we shall generalize our previous considerations [13] and discuss the SI scalar field behavior in the spacetime of dilaton black hole. In Sec.II we gave the analytic arguments concerning the intermediate and the very late-time behavior of SI scalar field in the background of the considered black hole. Then, in Sec.III we treated the problem numerically. Sec.IV will be devoted to a summary and conclusions.

\section{THE EINSTEIN-MAXWELL-DILATON EQUATIONS}

In this section we shall study the evolution of SI (massive) scalar field $\tilde{\psi}$ around a fixed background of electrically charged dilaton black hole. The wave equation for the field is given by

$$
\nabla_{\mu} \nabla^{\mu} \tilde{\psi}-m^{2} \tilde{\psi}^{2}=0
$$

where $m$ is assumed to be real.

The metric of the external gravitational field will be given by the static, spherically symmetric solution of Eqs. of motion derived from the low-energy string action (see e.g. [14]). The action has the form as follows:

$$
S=\int d^{4} x\left[R-2(\nabla \phi)^{2}-e^{-2 \phi} F^{2}\right]
$$

where $\phi$ is the dilatonic field and $F_{\alpha \beta}=2 \nabla_{[\alpha} A_{\beta]}$. The metric of electrically charged dilaton black hole [14] implies

$$
d s^{2}=-\left(1-\frac{2 M}{r}\right) d t^{2}+\frac{d r^{2}}{\left(1-\frac{2 M}{r}\right)}+r\left(r-\frac{Q^{2}}{M}\right)\left(d \theta^{2}+\sin \theta d \phi^{2}\right) .
$$

The event horizon is located at $r_{+}=2 M$. For the case of $r_{-}=\frac{Q^{2}}{M}$ we have another singularity but it can be ignored because of the fact that $r_{-}<r_{+}$. The dilaton field is given by $e^{2 \phi}=e^{-2 \phi_{0}}\left(1-\frac{r_{-}}{r}\right)$, where $\phi_{0}$ is the dilaton's value at $r \rightarrow \infty$. The mass $M$ and the charge $Q$ are related by the relation $Q^{2}=\frac{r_{+} r_{-}}{2} e^{2 \phi_{0}}$.

Defining the tortoise coordinate $y$, as $d y=\frac{d r}{\left(1-\frac{2 M}{r}\right)}$ one can rewrite (3) in the form

$$
d s^{2}=\left(1-\frac{2 M}{r}\right)\left[-d t^{2}+d y^{2}\right]+r\left(r-\frac{Q^{2}}{M}\right)\left(d \theta^{2}+\sin \theta d \phi^{2}\right) .
$$

For the spherical background each of the multipole of perturbation field evolves separetly so for the scalar field in the form $\tilde{\psi}=\sum_{l, m} \psi_{m}^{l}(t, r) Y_{l}^{m}(\theta, \phi) / R(r)$, one has the following equations of motion for each multipole moment

$$
\psi_{, t t}-\psi_{, y y}+V \psi=0
$$

where 


$$
V=\left[\frac{l(l+1)}{R^{2}}+\frac{R^{\prime \prime}}{R}\left(1-\frac{2 M}{r}\right)+\frac{2 M R^{\prime}}{r^{2} R}+m^{2}\right]\left(1-\frac{2 M}{r}\right) .
$$

By $R$ we denoted $R=\sqrt{r\left(r-\frac{Q^{2}}{M}\right)}$ and ' is the derivative with respect to the $r$-coordinate.

In order to analyze the time evolution of SI field in the background of dilaton black hole we shall use the spectral decomposition method [15]. The time evolution of SI scalar field is given by

$$
\psi(y, t)=\int d y^{\prime}\left[G\left(y, y^{\prime} ; t\right) \psi_{t}\left(y^{\prime}, 0\right)+G_{t}\left(y, y^{\prime} ; t\right) \psi\left(y^{\prime}, 0\right)\right]
$$

for $t>0$, where the Green's function $G\left(y, y^{\prime} ; t\right)$ is defined by

$$
\left[\frac{\partial^{2}}{\partial t^{2}}-\frac{\partial^{2}}{\partial y^{2}}+V\right] G\left(y, y^{\prime} ; t\right)=\delta(t) \delta\left(y-y^{\prime}\right)
$$

Our main task will be to find the black hole Green function. The first step in finding $G\left(y, y^{\prime} ; t\right)$ consists of reducing (8) to an ordinary differential equation. To do it one can use the Fourier transform [16]

$$
\tilde{G}\left(y, y^{\prime} ; \omega\right)=\int_{0^{-}}^{\infty} d t G\left(y, y^{\prime} ; t\right) e^{i \omega t}
$$

This transform is well defined if $\operatorname{Im} \omega \geq 0$, while the corresponding inverse transform has the form

$$
G\left(y, y^{\prime} ; t\right)=\frac{1}{2 \pi} \int_{-\infty+i \epsilon}^{\infty+i \epsilon} d \omega \tilde{G}\left(y, y^{\prime} ; \omega\right) e^{-i \omega t}
$$

for some positive number $\epsilon$.

The Fourier's component of the Green's function $\tilde{G}\left(y, y^{\prime} ; \omega\right)$ can be expressed in terms of two linearly independent solutions for homogeneous equation, namely

$$
\left(\frac{d^{2}}{d y^{2}}+\omega^{2}-V\right) \psi_{i}=0, \quad i=1,2
$$

The boundary conditions for $\psi_{i}$ are described by purely ingoing waves crossing the outer horizon $H_{+}$of the dilaton black hole $\psi_{1} \simeq e^{-i \omega y}$ as $y \rightarrow-\infty$ while $\psi_{2}$ should be damped expotentially at $i_{+}$, namely $\psi_{2} \simeq e^{-\sqrt{\omega^{2}-m^{2}} y}$ at $y \rightarrow \infty$.

In order to find $\psi_{i}$ we consider the wave Eq.(11) of SI scalar field and transform it in such a way that [7] the Coulomb and Newtonian $(1 / r)$ potentials will dominate at large distances. One can introduce an auxiliary variable $\xi$ in such a way that $\xi=A(r) \psi$, where $A(r)^{2}=1-\frac{2 M}{r}$. So in terms of the variable $\xi$ Eq. (11) can be rewritten as follows:

$$
\frac{d^{2} \xi}{d r^{2}}-\frac{A_{, r r}}{A} \xi+\frac{1}{A^{4}}\left[\omega^{2}-A^{2}\left(\frac{l(l+1)}{R^{2}}+\frac{R^{\prime \prime}}{R} A(r)^{2}+\frac{2 M R^{\prime}}{r^{2} R}+m^{2}\right)\right] \xi=0
$$

Now we expand Eq.(12) in power series of $\frac{M}{r}$ and $\frac{Q}{r}$, neglecting terms of order $O\left(\frac{\alpha}{r^{n \geq 2}}\right)$. Thus we arrive at the following expression:

$$
\frac{d^{2} \xi}{d r^{2}}+\left[\omega^{2}-m^{2}+\frac{4 M \omega^{2}-2 M m^{2}}{r}-\frac{l(l+1)}{r^{2}}\right] \xi=0
$$

If one further assumes that the observer and the initial data are in the region where $r \leq \frac{M}{(M m)^{2}}$ and one shall be interested in the intermediate asymptotic behavior of SI scalar field $r \leq t \leq \frac{M}{(M m)^{2}}$, then we get 


$$
\frac{d^{2} \xi}{d r^{2}}+\left[\omega^{2}-m^{2}-\frac{l(l+1)}{r^{2}}\right] \xi=0
$$

The above approximation neglects the backscattering of the SI field from asymptotically far regions. As in Ref. [8] in our case of the scalar field perturbations on dilaton black hole we get the dependence on the field's parameter (the mass of the field). These perturbations do not depend on the spacetime parameters $(Q$ and $M)$.

The procedure of getting the solution of Eq.(14) is the same as described in [8] so we refer the reader to this work. We only write the final form of the $\psi$ dependence. The intermediate asymptotic behavior of the SI field at a fixed radius has the form 8

$$
\psi \sim t^{-\left(l+\frac{3}{2}\right)} \sin m t
$$

while the intermediate behavior of the considered field at dilaton black hole outer horizon $H_{+}$is dominated by

$$
\psi \sim v^{-\left(l+\frac{3}{2}\right)} \sin m t
$$

They are dominated by an oscillatory power law tails.

Recently the late-time behavior of the SI field in Schwarzschild background was studied in Ref. [12]. Following their footsteps we shall write the wave Eq. (14) using the non-dimensional variable $x=r / 2 M$. Then we reach to the analytically tractable equation of the form

$$
\frac{d^{2} \psi}{d x^{2}}+\frac{1}{x(x-1)} \frac{d \psi}{d x}+\left[\frac{4 M^{2} \omega^{2} x^{2}}{(x-1)^{2}}-\frac{l(l+1)}{x(x-1)}-\frac{1}{x^{2}(x-1)}+\frac{4 m^{2} M^{2} x}{x-1}\right] \psi+O\left(\frac{Q}{M}\right)=0 .
$$

The procedure of getting the solution to Eq.(17) was presented in details in Ref. [12]. Here we quote the main conclusion, namely the smaller value of $m M$ is the later the $t^{-5 / 6}$ tail begins to dominate. It is true for the range of parameter $m t \gg \frac{1}{(m M)^{2}}$. For $m t \gg m M$ it was found that the larger value of $m M$ is the later the $t^{-5 / 6}$ tail begins to dominate. Of course these conclusion are valid for small $Q$. In the next section we investigate this problem numerically.

\section{NUMERICAL RESULTS}

We numerically analyzed Eq. (5) using method described in [4]. We transformed Eq. (5) into (u,v) coordinates

$$
4 \psi_{, u v}+V \psi=0
$$

and solved it on uniformly spaced grid using explicit difference scheme. As was pointed out previously the late time evolution of a massive field is independent of the form of the initial data. To start our calculation we used a Gaussian pulse of the form as follows:

$$
\psi(u=0, v)=A \exp \left(-\frac{\left(v-v_{0}\right)^{2}}{\sigma^{2}}\right)
$$

Linearity of the Eq.(5) leaves us a freedom to choose the amplitude $A$, and for our purposes we used $A=1$. The rest of the initial field profile parameters were $v_{0}=50$ and $\sigma=2$. The black hole mass and charge are set equal to 
$M=0.5$ and $Q=0.45$, respectively, and mass of the field is $m=0.01$. We have studied the behavior of the field $\psi$ on two hypersurfaces: the future timelike infinity, $i_{+}$(approximated in our calculations by the field at fixed radius $y=50$ ), and the black hole future horizon $H_{+}$(approximated by the field on the null surface $u=10^{4}$ ). First, we have examined $l=0$ modes. The results are presented in Fig. 1. After initial period dominated by prompt contribution and quasi-normal ringing the field establishes a definite oscillatory pattern. The amplitudes of the field decrease according to power-law with index -1.51 for both $i_{+}$(top curve) and $H_{+}$. These exponents were calculated by fitting a straight line to the oscillations extrema within the time range $2 \times 10^{3} \div 7 \times 10^{3}$ to avoid influence of the initial perturbation and the late time tails. Analytically estimated from Eqs.(15)-(16) value for the index is -1.5 providing excellent agreement with numerical results. The period of the oscillations (the distance between two neighbor maxima) is $314 \pm 0.5$ which means less than $0.2 \%$ discrepancy from analytically expected period $T=\pi / m \simeq 314.5$

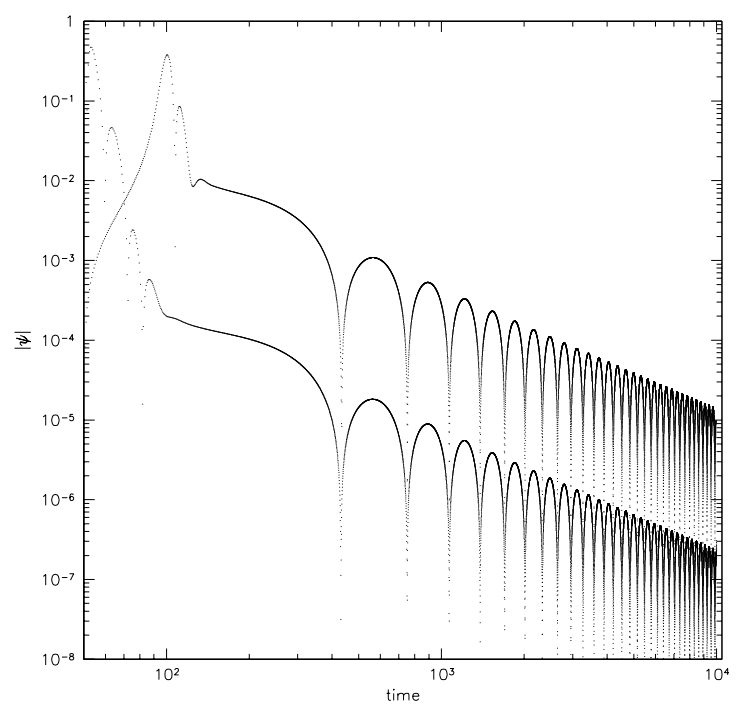

FIG. 1. Evolution of the field $|\psi|$ for $l=0, M=0.5, Q=0.45$ and $m=0.01$. Upper curve represents the field at fixed radius $y=50$ (approximation of the future timelike infinity $i_{+}$) as a function of $\mathrm{t}$. Lower curve represents the field at $u=10^{4}$ (approximation of the black hole future horizon $H_{+}$) as a function of $v$. The power-law exponents are -1.51 for both $i_{+}$and $H_{+}$. The period of the oscillations is $T=\pi / m \simeq 314.5$ to within $0.2 \%$.

Secondly, we considered the dependence of the SI scalar field on the multipole index $l$. The results are presented in Fig. 2. The behavior of the field on the future timelike infinity is calculated for $l=0,1,2$ and 3 . The numerical values of the power-law exponents are $-1.51,-2.52,-3.53$, and -4.51 , respectively. According to Eq.(15) these exponents equal to $-1.5,-2.5,-3.5$, and -4.5 , thus again the agreement between numerical calculations and analytical predictions is excellent. The oscillations period is $T=\pi / m \simeq 315$ to within $0.5 \%$, in agreement with the predicted value. 


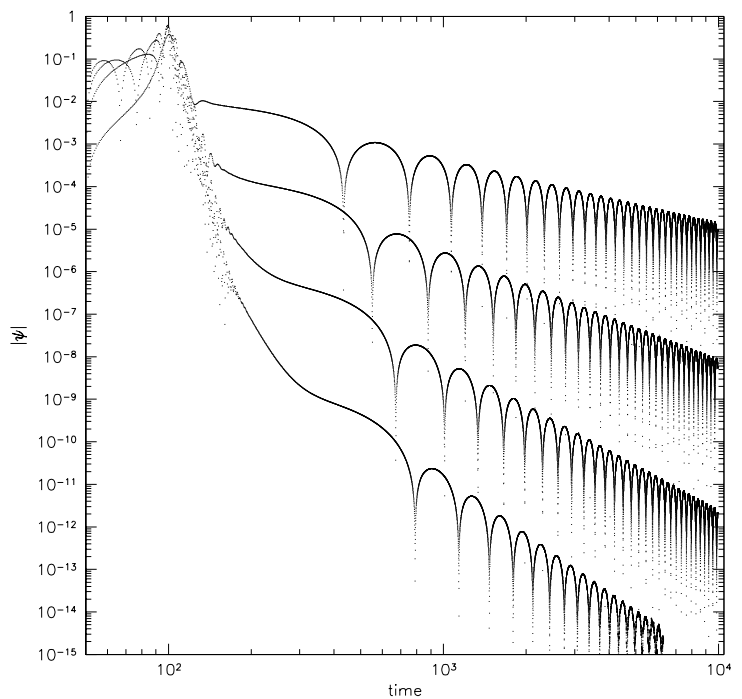

FIG. 2. Evolution of the field $|\psi|$ on the future timelike infinity $i_{+}$(approximated by $\psi(y=50, t)$ ) for different multipoles $l=0,1,2$ and 3 . The power-law exponents are $-1.51,-2.52,-3.53$, and -4.51 , respectively. The period of the oscillations is 314.5 to within $0.5 \%$ (for the worst case of $l=3$ ).

Finally, we have checked the late-time behavior of the field. In Fig. 3 we present the field on the future timelike infinity $i_{+}$and on the black hole horizon $H_{+}$as a function of time for $l=0$ mode (for presentation purposes we plot only maxima of the oscillations). As in [10] our numerical scheme reveals the fact that after the intermediate phase of the power-law decay the amplitude of the field decreases slower than any power-law. In the very late approximation the change of the tail will be result of the dominant backscattering due to the spacetime curvature. This effect is beyond the flat space approximation as was remarked in [1].

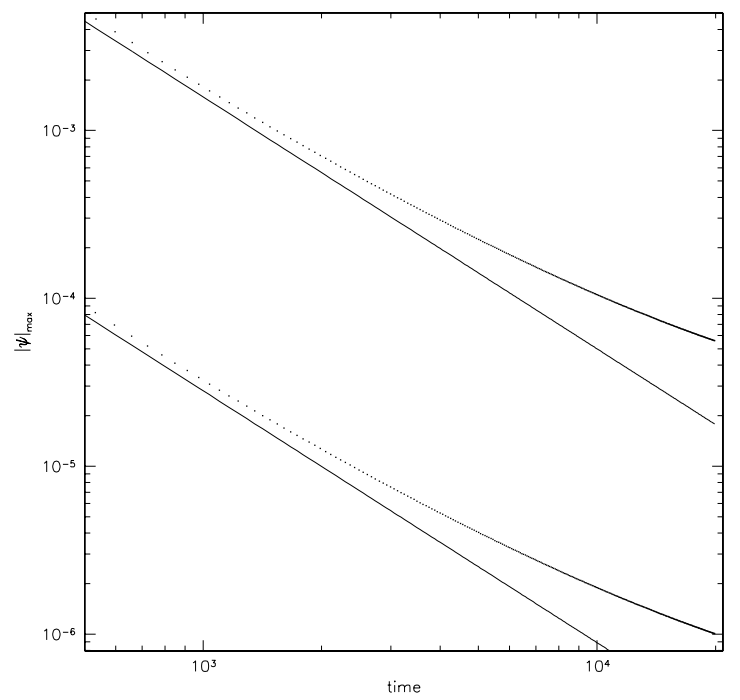

FIG. 3. Late-time behavior of the field $|\psi|_{\max }$ on dilaton black hole. The mass of the field $m=0.05$, the upper curve represents the field at future timelike infinity $(y=50)$ as a function of time $t$. The lower curve along the black hole horizon as a function of $v$. The period of oscillations is 63.0 to within $0.8 \%$. The thin solid lines have slopes equal to -1.5 . 
Further, we fix mass and charge of dilaton black hole and change the mass of the SI scalar fields. We have noted the relation between mass $m$ and the another pattern of decay; namely, the smaller $m$ is the later begins to dominate this pattern. We remark that analogical relation was obtained from analytical considerations when the charge of dilaton black hole was assumed to be small. In our numerical simulations we get rid of this assumption.

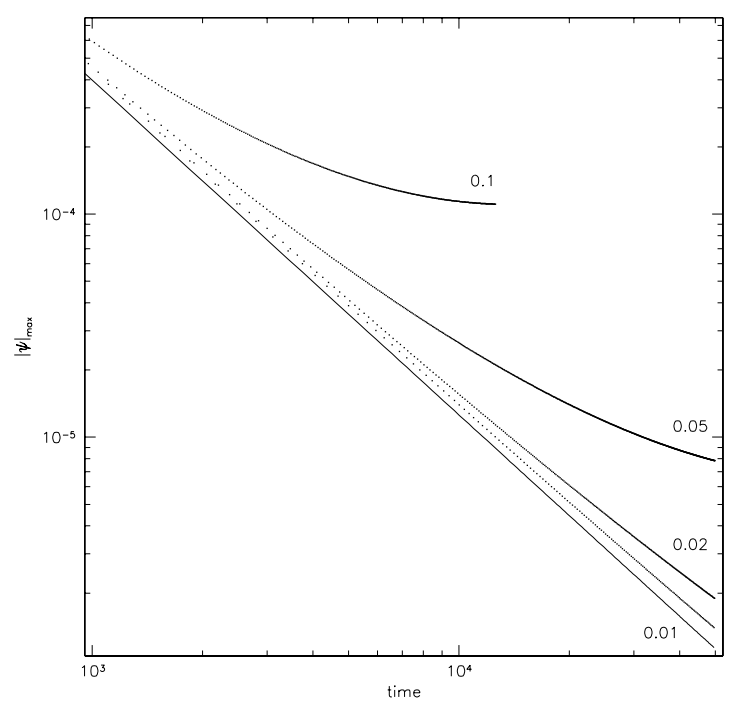

FIG. 4. Late-time behavior of the SI scalar field $|\psi|_{\max }$ (maxima of the oscillations) at future timelike infinity for different masses $m$ as a function of time $t$. The thin solid line has slope equals to -1.5 , mass of dilaton black hole $M=0.5$ and charge $Q=0.45$.

\section{CONCLUSIONS}

In our work we have investigated the intermediate asymptotic behavior of SI scalar field in the background of dilaton black hole being the spherical symmetric solution of the so-called low-energy string theory 14. As was revealed the late-time behavior of the charged massless perturbations depended on the spacetime parameters $M, Q$, while the intermediate asymptotic behavior of SI field depended only on the field's mass $m$. The numerical scheme has shown that at the very late-time the inverse power-law decay is replaced by another kind of the decay process, slower than any power-law. From our numerical calculations we have established that if one fixed the mass and charge of dilaton black hole $M$ we get the relation: the smaller SI field mass is, the later begins the process. This very late-time behavior needs further analytic studies. The first step towards it was done in 11, 12 studying nearly extreme RN and Schwarzschild solutions. We have found that in the case of small $Q$ comparing to $M$, our wave equation reduce to the form studied in [12]. Thus, it can be deduced that the asymptotic late-time behavior of SI scalar field is of the form of the oscillatory tail with the decay rate $t^{-5 / 6}$, which is identical with that in the nearly extreme RN or Schwarzschild background.

Another problem for further investigations is the problem of late-time behavior of massless and SI scalar fields in the spacetime of extremal dilaton black hole. We hope to return to this problem elsewhere. 
[1] P.O.Mazur, hep-th 0101012 (2001),

M.Heusler, Black Holes Uniqueness Theorems (Cambridge University Press, Cambridge, England, 1996),

K.S.Thorne, Black Holes and Time Warps (W.W.Norton and Company, New York, 1994).

[2] E.Poisson and W.Israel, Phys. Rev. D 41, 1796 (1990).

[3] R.H.Price, Phys. Rev. D 5, 2419 (1972).

[4] C.Gundlach, R.H.Price and J.Pullin, Phys. Rev. D 49, 883 (1994).

[5] J.Bicak, Gen. Rel. Grav. 3, 331 (1972).

[6] S.Hod and T.Piran, Phys. Rev. D 58, 024017 (1998).

[7] S.Hod and T.Piran, Phys. Rev. D 58, 024018 (1998).

[8] S.Hod and T.Piran, Phys. Rev. D 58, 024019 (1998).

[9] L.M.Burko, Abstracts of plenary talks and contributed papers, 15th International Conference on General Relativity and Gravitation, Pune, 1997, p.143, unpublished.

[10] S.Hod and T.Piran, Phys. Rev. D 58, 044018 (1998).

[11] H.Koyama and A.Tomimatsu, Phys. Rev. D 63, 064032 (2001).

[12] H.Koyama and A.Tomimatsu, gr-qc 0103086 (2001).

[13] R.Moderski and M.Rogatko, Phys. Rev. D 63, 084014 (2001).

[14] D.Garfinkle, G.T.Horowitz, and A.Strominger, Phys. Rev. D 43, 3140 (1991),

C.F.E.Holzhey and F.Wilczek, Nucl. Phys. B 380, 447 (1992).

[15] E.W.Leaver, Phys. Rev. D 34, 384 (1986).

[16] N.Anderson, Phys. Rev. D 51, 353 (1995). 